\title{
The factors affecting the redisplacement in distal radius fractures
}

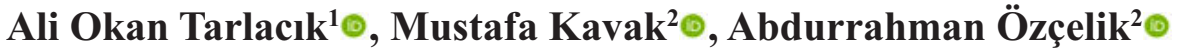 \\ ${ }^{1}$ Department of Orthopedics and Traumatology, Eskişehir City Hospital, Eskişehir, Turkey \\ ${ }^{2}$ Department of Orthopedics and Traumatology, Eskişehir Osmangazi University School of Medicine, Eskişehir, Turkey
}

\begin{abstract}
Objectives: Many instability criteria have been described in the literature to predict loss of reduction in distal radius fractures. However, the effect of the column location of the fracture on loss of reduction has not been investigated. The aim of this study is to investigate the effect of fracture column location and other radiological parameters on the loss of reduction of the distal radius fractures.

Methods: A total of 106 patients who were treated conservatively for displaced distal radius fractures were included in the study. The average age of the patients included in the study is 54.9 years (range: 18-91 years). Anteroposterior and lateral radiographs of the wrist were taken in each patient at the time of first admission, immediately after reduction and casting, and at the $1^{\text {st, }} 2^{\text {nd }}$ and $6^{\text {th }}$ weeks. Intraarticular fracture were evaluated by computed tomography (CT). Radial length, volar tilt angle and column location of the fracture were obtained by evaluating the radiographs and $\mathrm{CT}$. The effects of post-reduction radiological parameters and column location of the fracture on loss of reduction were analyzed.
\end{abstract}

Results: Reduction loss was detected in $23(21.7 \%)$ of 106 patients. Metaphyseal fracture in $83(78.3 \%)$ patients, intermediate volar column fracture in $76(71.7 \%)$ patients, intermediate dorsal column fracture in 86 $(81.1 \%)$ patients, ulnar column fracture in $52(49.1 \%)$ patients and radial column fracture in $25(23.6 \%)$ patients were determined. It was observed that having a fracture in the ulnar column or radial column caused a significant loss in radial length $(p<0.05)$. Metaphyseal and the intermediate column fractures did not make a statistically significant difference in reduction loss.

Conclusions: Column location of the fracture can also be used to predict loss of reduction in the conservative treatment of distal radius fractures.

Keywords: distal radius fracture, instability, loss of reduction, redisplacement, column theory

D istal radius fractures account for approximately one-fifth of fractures treated in emergency departments [1]. The first treatment of patients with distal radius fractures is usually conservative treatment consisting of closed reduction and plaster immobilization $[2,3]$. However, loss of reduction occurs in up to $64 \%$ of patients after closed reduction [4].
It is a widely accepted opinion that surgical fixation will benefit patients in distal radius fractures with loss of reduction other than acceptable parameters $(>$ $10^{\circ}$ dorsal angulation, radial shortening $>3 \mathrm{~mm}$ or intra-articular step-off) (American Academy of Orthopaedic Surgeons Board of Directors, 5 December 2009), considering the patient-related factors. 
Lafontaine et al. [5] has defined five basic criteria to identify patients with unstable distal radius fractures: dorsal angulation exceeding $20^{\circ}$ at presentation; dorsal comminution; extension of the fracture into the radiocarpal joint; an associated ulnar fracture; and age over 60 years. In cases where three or more of these criteria are present, the fracture is considered unstable [5]. After this study, many studies were conducted to determine the instability criteria in distal radius fractures. Some of them confirmed these five criteria, while others rejected them $[6,7]$. An other study identified different radiological and clinical risk factors and developed scoring systems to predict loss of reduction [8]. As a result, the factors determined about instability in the literature are; patient age 60 or above, metaphyseal comminution, dorsal angulation greater than $20^{\circ}$, shortening of radius greater than $5 \mathrm{~mm} /$ positive ulnar variance, associated ulnar fracture, severe osteoporosis, radial inclination less than $15^{\circ}, 2 \mathrm{~mm}$ joint stepping and widening of the joint surface more than $50 \%$ $[5,8,9]$. Although there are many risk factors used in the literature to predict the loss of reduction in distal radius fractures, the evidence regarding them is still limited [10]. In addition, the effect of the column lo- cation of the fracture on loss of reduction has not been investigated. Therefore, we determined the main research question as ' Does the column localization of the fracture have an effect on reduction loss in conservatively treated distal radius fractures?'. The primary aim of this study is to investigate the effect of fracture column location on the loss of reduction of the distal radius fractures. The secondary aim of this study is to investigate the effects of age, post-reduction radiological parameters on reduction loss in patients with conservatively treated distal radius fractures. The hypothesis of the present study is 'The column localization of the fracture can be used to assess the stability of distal radius fractures.

\section{METHODS}

The patients were informed verbally and written with a consent form describing the procedure. The study was conducted according to the Helsinki Declaration and approved by Local Commitee of Medical Ethics for human studies (03.08.2018/10).

One hundred and six out of a total of 123 patients,

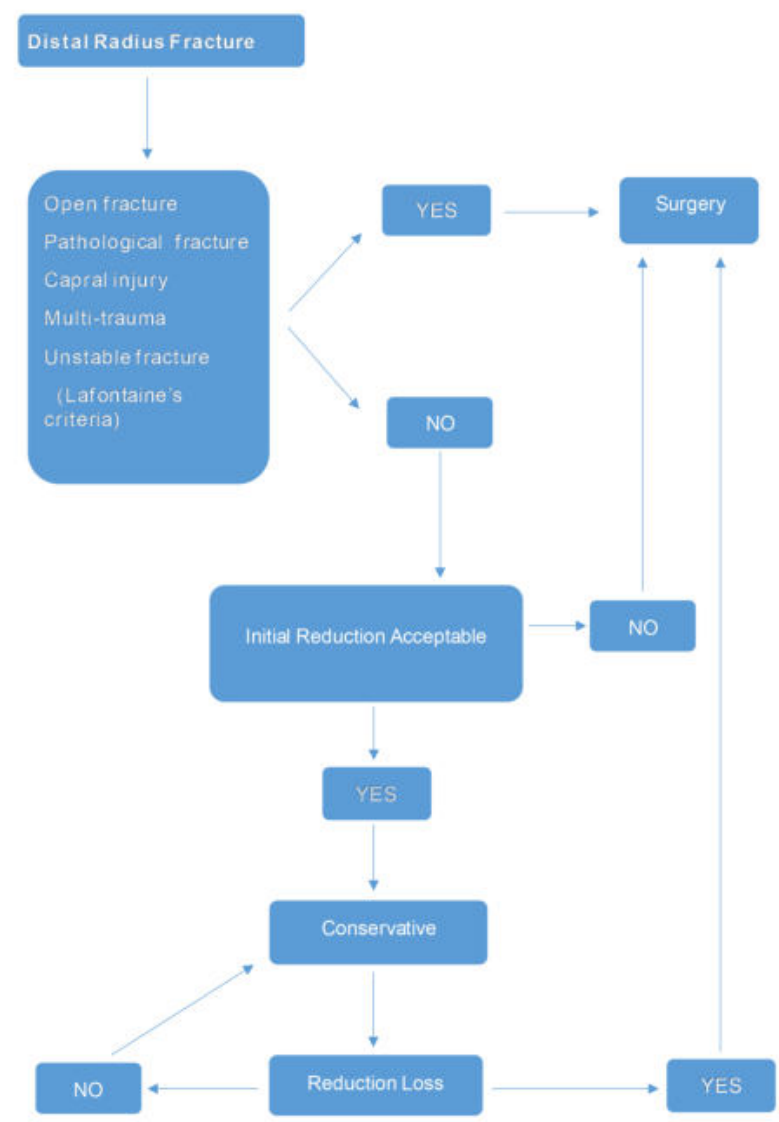

Fig. 1. Flowchart of patient selection and treatment algorithm. 
75 females and 31 males, who were treated conservatively for distal radius fractures in our clinic between May 2018 and October 2019 were included in the study. Seventeen patients who were lost to follow up were excluded from the study. Patients with multiple fractures, pathological fractures, open fractures, carpal injuries and unstable fractures (according to Lafontaine's criteria [5]) were not included in the study. In addition, patients who could not achieve acceptable reduction after the first reduction were not included in the study (Fig. 1). The average age of the patients included in the study is 54.9. (18-91 range). Closed reduction under sedation and long arm circular cast were applied to all patients. Anteroposterior and lateral direct radiographs of the wrist were taken in each patient at the time of first admission, immediately after reduction and casting, at the $1^{\text {st }}$ week, $2^{\text {nd }}$ week and $6^{\text {th }}$ week. Computed tomography (CT) was performed to determine the column localization of the fracture only in intra-articular fractures. Patients were followed until union was observed. Radial length and volar tilt angle were measured by examining all direct graphs. In addition, the distal forearm was divided into five basic anatomical regions, considering the column theory [11] and Melone [6] classification. These regions were; the extrarticular metaphyseal part was classified as metaphyseal region, the area lateral to the Lister's tubercle containing the scaphoid fossa as the radial column, the area medial to the Lister's tubercle con- taining the lunate fossa as the intermediate column, and the distal ulna as the ulnar column. In addition, the intermediate column was divided into two as volar and dorsal regions. Considering these five main regions, graphies and CT scans were evaluated and the column locations of the fractures were determined (Fig. 2). All the measurements were conducted by one independent orthopedic surgeon.

In the measurements made on the radiographs obtained during the follow-up of the patients; according to AAOS criteria, patients with a dorsal angulation of the volar tilt angle over 10 degrees and shortening of more than $3 \mathrm{~mm}$ in radial length were evaluated as reduction loss [12].

\section{Statistical Analysis}

The effects of age, fracture column location, radial length and volar tilt after first reduction parameters on reduction loss were analyzed. Shapiro Wilk test was used to investigate the compatibility of the data to normal distribution, the two-way repeated measures ANOVA test was used for repeated measures, the McNemmar test was used for the comparison before and after the analysis of the cross tables, and the standard SPSS for Windows (version 21.0, IBM Corp., Armork, New York) was used for the analysis. $P<0.05$ value was accepted as the criterion for statistical significance.
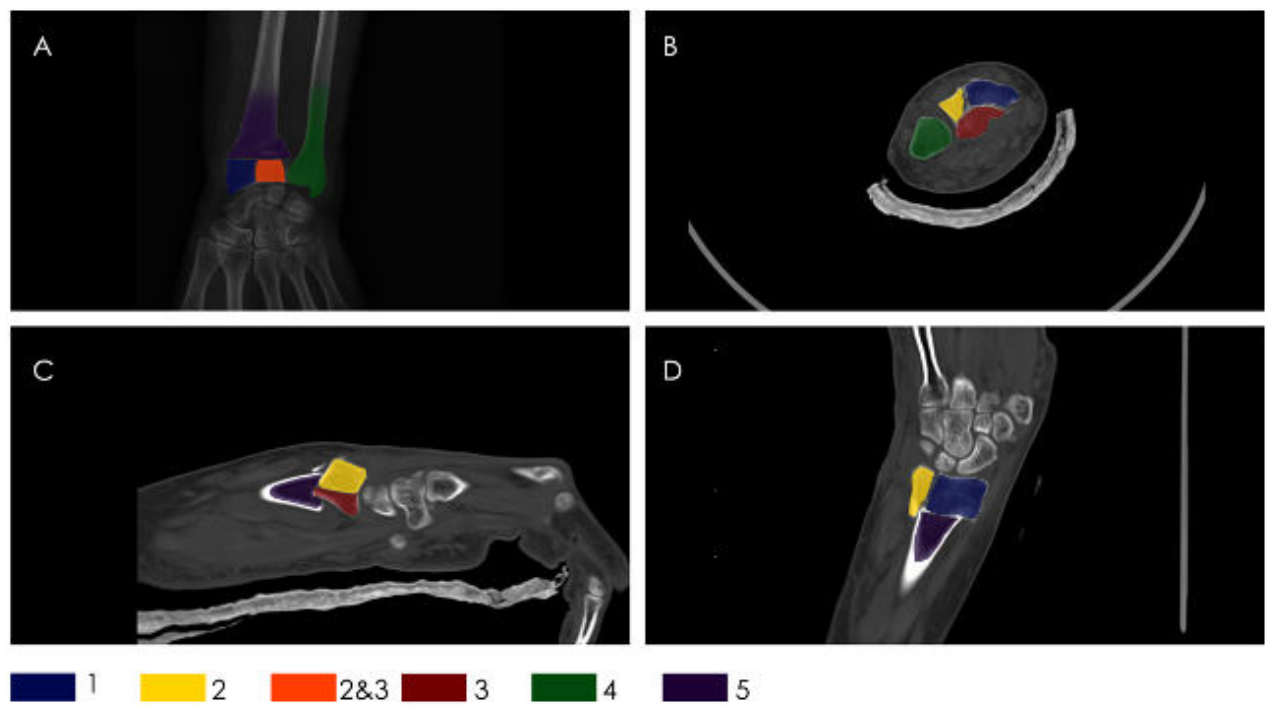

Fig. 2. (A) AP view distal radius X-Ray, (B) Axial view distal radius CT, (C): Sagittal view distal radius CT, (D) Coronal view distal radius CT. Distal radius column illustration: 1) Blue $=$ Radial column, 2) Yellow $=$ Dorsal intermediate column, 2\&3) Orange $=$ Intermediate column, 3) Red $=$ Volar intermediate column, 4) Green $=$ Ulnar column, and 5) Purple $=$ Metaphyseal region. 


\section{RESULTS}

Examining the anatomical location of 106 distal radius fractures, it was determined that $83(78.3 \%)$ patients had metaphyseal fractures, 76 (71.7\%) patients had intermediate volar colon fractures, $86(81.1 \%)$ patients had intermediate dorsal colon fractures, 52 (49.1\%) patients had ulnar colon fractures and 25 (23.6\%) patients had radial column fracture. In the first radiographs after reduction, the radial height was detected as an average of $12.35 \mathrm{~mm}(3.92-20.68 \mathrm{~mm})$, and the average volar tilt angle was $5.23^{\circ}(-17.50$ $\left.21.90^{\circ}\right)$. In the last control radiographs of the patients, the radial height was detected as an average of 9.25 $\mathrm{mm}(-1.12-18.80 \mathrm{~mm})$, and the mean volar tilt angle was $1.55^{\circ}\left(-22.10-25.10^{\circ}\right)($ Table 1$)$.

Reduction loss was detected in $23(21.7 \%)$ of 106 patients. It was observed that $19(17.9 \%)$ of them had reduction loss in radial length and in 4 (3.8\%) of them in volar tilt. There was no statistically significant difference between reduction loss and age (OR; 95\% confidence interval (CI): 0.224 [0.936-1.022]). There was no statistically significant difference between reduction loss and after reduction measurements of the radial length (OR; 95\% CI: 0.935 [0.734-1.191]) and volar tilt angle (OR; 95\% CI: 0.979 [0.893-1.074]). It was found that ulnar (OR; 95\% CI: 6.993 [2.519.607]) or radial (OR; 95\% CI: 3.871 [1.264-11.904]) column fractures of the distal radius causes significant loss of radial length $(p<0.05)$. There was no statistically significant relationship between the fractures in the metaphyseal region (OR; 95\% CI: 0.253 [0.0511.262]), intermediate volar column (OR; 95\% CI: 1.012 [0.303-3.381]) and intermediate dorsal column (OR; 95\% CI: 1.874 [0.486-7.224]) with radial length $(p>0.05)$ (Table 2). Among the criteria investigated, no statistically significant parameter effective in volar tilt loss was observed ( $p>0.05$ ) (OR; 95\% CI: 0.169).

\section{DISCUSSION}

In this study, in conservatively treated distal radius fractures, the effect of the fracture's column location on loss of reduction was investigated and it was concluded that the radial length loss was greater in fractures involving the radail and ulnar column. The rate of reduction loss in conservatively treated distal radius fractures is $9-32 \%$ [13]. Factors causing reduction loss in conservatively treated distal radius fractures have been investigated in many studies in the literature. The colon theory has been defined as the concept of stable fixation in the surgical treatment of distal radius fractures. However, the effect of the column theory on reduction loss in conservative treatment has not been examined in the literature.

In order to classify distal radius fractures, there are more than twenty classification systems that have been

Table 1. Radiographic parameters in each session

\begin{tabular}{lcc}
\hline & $\begin{array}{c}\text { After Reduction } \\
\text { (min-max) }\end{array}$ & $\begin{array}{c}\text { Final Follow-up } \\
\text { (min-max) }\end{array}$ \\
\hline Radial Length & $12.35 \mathrm{~mm}(3.92-20.68)$ & $9.25 \mathrm{~mm}(-1.12-18.8)$ \\
Volar Tilt & $5.23^{\circ}(-17.5-21.9)$ & $1.55^{\circ}(-22.1-25.1)$ \\
\hline
\end{tabular}

Table 2. Fracture column location with radial length displacement on logistic regression analysis

\begin{tabular}{lcccc}
\hline & Odds Ratio & $\begin{array}{c}\text { 95\% CI for OR } \\
\text { Lower }\end{array}$ & $\begin{array}{c}\text { 95\% CI for } \\
\text { OR Upper }\end{array}$ & $p$ value \\
\hline Metaphyseal Fracture & 0.253 & 0.051 & 1.262 & 0.094 \\
Intermediate Dorsal Column Fracture & 1.874 & 0.486 & 7.224 & 0.362 \\
Intermediate Volar Column Fracture & 1.012 & 0.303 & 3.381 & 0.985 \\
Ulnar Column Fracture & 6.993 & 2.5 & 19.607 & $\mathbf{0 . 0 0 0 1}$ \\
Radial Column Fracture & 3.871 & 1.264 & 11.904 & $\mathbf{0 . 0 1 8}$ \\
\hline
\end{tabular}


defined so far according to the surgeon's name, the mechanism of the fracture, the area of the fracture, and the stability. Although classifications are useful in academic use, they remain inadequate due to the deficiencies in guiding the treatment method and the inability to form a common consensus. There are many articles comparing surgical and conservative treatment. Arora et al. [2] compared the results of surgical and conservative treatment options in patients over 65 years of age with distal radius fractures and found that there was no difference between the two groups in range of motion and pain level. In the same study, they showed that surgical treatment had better results in grip strength, volar tilt angle measurements and radial length measurement. They stated that conservative treatment had better results in complication rates. Zengin et al. [14], in their study comparing volar plating and plaster treatment in patients aged 60 and over with AO type c distal radius fractures, reported that the surgical method yielded better grip strength and radiological results and there was no statistically difference between clinical and functional results. Surgical and conservative treatment options were also compared in terms of cost. In their study, Shauver et al. [15] showed that the cost of conservative treatment was much lower when they compared the treatment costs, although surgery was preferred more in the treatment of distal radius fractures in elderly patients. Toon et al. [16] compared open reduction and internal fixation with conservative treatment in the treatment of distal radius fractures and found that there was a 37-times difference between these two methods in the treatment cost. For this reason, finding certain criteria for conservative treatment in distal radius fractures will provide an economically important advantage.

The concept of stability gains importance in the conservative treatment of distal radius fractures. There are many studies investigating the instability criteria in distal radius fractures [17]. It is claimed that; patient age 60 or above, comminution of metaphyseal dorsal cortex, dorsal angulation greater than $20^{\circ}$, shortening of radial length more than $5 \mathrm{~mm} /$ positive ulnar variance, associated ulnar fracture, severe osteoporosis, radial inclination less than $15^{\circ}$, joint stepping of more than $2 \mathrm{~mm}$ and an enlargement of more than $50 \%$ in the joint face are important in instability $[12,18]$. Leone et al. [19] claimed that the degree of radial shortness and volar tilt angle are associated with early instability, radial inclination, age, shortening of the radial length and volar tilt angle are associated with late instability. Nesbitt et al. [9] argued that there is a 50\% risk of loss of reduction in patients over the age of 58 . In our study, there was no significant difference between ages in terms of loss of reduction. In many studies, shortening of the radial length is stated to be the most important radiological instability factor affecting the prognosis, so correction of the radial length should be the primary goal $[20,21]$. Volar tilt angle is also claimed to be one of the important radiological instability criteria [22]. Perugia et al. [23] reported that volar tilt angle and ulnar variance are the most important radiological parameters that need to be corrected in order to achieve good functional results in patients with distal radius fractures. In addition, associated ulnar styloid fracture is an important radiological parameter that increases the possibility of functional limitation [24]. Lyu et al. [25] investigated the risk factors causing radial shortening in patients with distal radius fractures who were followed up conservatively. They stated age, time between injury and reduction, fracture classification and early weight bearing as risk factors that will cause shortening of the radial length. Similarly, in our study, it was observed that fractures in the radial and ulnar colon created a significant difference in radial length. However, it was determined that a fracture in any column does not cause a statistically significant change on the volar tilt angle.

There are very few theories regarding clinical evaluation with respect to the anatomical columns in the distal radial region. The column concept was defined for surgical fixation stability but was omitted in the stability assessment for conservative management of distal radius fractures. In the examination performed over the columns in our study, it was observed that radial and ulnar column involvement were important risk factors for loss of reduction. There are studies in the literature showing that the ulna styloid fracture accompanying the distal radius fracture causes instability [24]. Similarly, in our study, it has been shown that ulna styloid fracture, therefore, fracture in the ulnar column is one of the effective factors in reduction loss.

The radius and ulna styloid regions in the radial and ulnar columns are the attachment sites of the important ligaments that provide wrist stability (radial collateral ligament, radioscaphocapitate ligament, ra- 
diolunotriquetral ligament, ulnar collateral ligament, triangular fibrocartilage complex). In our study, it has been shown that fractures in these regions are associated with subsequent loss of reduction by causing radial length loss. Again, in a recent study, it was shown that radial shortening during conservative follow-up is a parameter that can be used to predict poor outcomes [25]. Further investigations are required between distal radius fractures and fracture instability, including wrist ligaments.

\section{Limitations}

Our study has a few limitations. Most importantly, our sample size is small and our study is retrospective. Besides, the initial displacement degree of the fracture is ignored. Another limitation is that measurements are made by a single independent surgeon. The subject that we want to focus on in our study is the relationship between the colon theory and the loss of reduction in the conservative treatment of distal radius fractures, which has not been studied in the literature, so other factors may have been ignored.

\section{CONCLUSION}

Column location of the fracture can also be used to predict loss of reduction in the conservative treatment of distal radius fractures. In our study, a significant relationship between radial length loss and radial and ulnar colon location of the fracture was demonstrated.

\section{Authors' Contribution}

Study Conception: AÖ; Study Design: AÖ; Supervision: AÖ; Funding: Osmangazi University Scientific Research Proect Unit; Materials: AOT; Data Collection and/or Processing: AOT; Statistical Analysis and/or Data Interpretation: AOT, MK; Literature Review: MK; Manuscript Preparation: MK and Critical Review: MK, AÖ.

\section{Conflict of interest}

The authors disclosed no conflict of interest during the preparation or publication of this manuscript.

\section{Financing}

This project was supported by Eskişehir Os- mangazi University Scientific Research Project Unit. The study sponsor was not involved in data collection or in data analysis and did not influence data interpretation. The sponsor did not decide where the manuscript is submitted.

\section{Acknowledgements}

The figures were prepared by Ms. Tugba KAVAK.

\section{REFERENCES}

1. MacDermid JC, Roth JH, Richards RS. Pain and disability reported in the year following a distal radius fracture: a cohort study. BMC Musculoskelet Disord 2003;4:24.

2. Arora R, Lutz M, Deml C, Krappinger D, Haug L, Gabl M. A prospective randomized trial comparing nonoperative treatment with volar locking plate fixation for displaced and unstable distal radial fractures in patients sixty-five years of age and older. J Bone Joint Surg Am 2011;93:2146-53.

3. Kumar S, Penematsa SR, Sadri M, Deshmukh SC. How many clinic visits does it take to treat distal radial fractures? Int Orthop 2008;32:91-6.

4.Makhni EC, Ewald TJ, Kelly S, Day CS. Effect of patient age on the radiographic outcomes of distal radius fractures subject to nonoperative treatment. J Hand Surg 2008;33:1301-8.

5. Lafontaine M, Hardy D, Delince P. Stability assessment of distal radius fractures. Injury 1989;20:208-10.

6. Melone CP Jr. Articular fractures of the distal radius. Orthop Clin North Am 1984;15:217-36.

7. Tahririan MA, Javdan M, Nouraei MH, Dehghani M. Evaluation of instability factors in distal radius fractures. J Res Med Sci 2013;18: 892-6.

8. Mackenney PJ, McQueen MM, Elton R. Prediction of instability in distal radial fractures. J Bone Joint Surg Am 2006;88:1944-51.

9. Nesbitt KS, Failla JM, Les C. Assessment of instability factors in adult distal radius fractures. J Hand Surg Am 2004;29:112838.

10. Walenkamp MM, Aydin S, Mulders MA, Goslings JC, Schep NW. Predictors of unstable distal radius fractures: a systematic review and meta-analysis. J Hand Surg Eur Vol 2016;41:501-15. 11. Rikli DA, Regazzoni P. Fractures of the distal end of the radius treated by internal fixation and early function. A preliminary report of 20 cases. J Bone Joint Surg Br 1996;78:588-92.

12. Lichtman DM, Bindra RR, Boyer MI, Putnam MD, Ring M, Slutsky DJ, et al. Treatment of distal radius fractures. J Am Acad Orthop Surg 2010;18:180-9.

13. Jung HW, Hong H, Jung HJ, Kim JS, Park HY, Bae KH, et al. Redisplacement of distal radius fracture after initial closed reduction: analysis of prognostic factors. Clin Orthop Surg 2015; 7:377-82.

14. Zengin EC, Ozcan C, Aslan C, Bulut T, Sener M. Cast immobilization versus volar locking plate fixation of $\mathrm{AO}$ type $\mathrm{C}$ distal radial fractures in patients aged 60 years and older. Acta 
Orthop Traumatol Turc 2019;53:15-8.

15. Shauver MJ, Clapham PJ, Chung KC. An economic analysis of outcomes and complications of treating distal radius fractures in the elderly. J Hand Surg Am 2011;36:1912-18.

16. Toon DH, Premchand RAX, Sim J, Vaikunthan R. Outcomes and financial implications of intra-articular distal radius fractures: a comparative study of open reduction internal fixation (ORIF) with volar locking plates versus nonoperative management. J Orthop Traumatol 2017; 18:229-34.

17. Wu YS, Yang J, Xie LZ, Zhang JY, Yu XB, Hu W, et al. Factors associated with the decision for operative versus conservative treatment of displaced distal radius fractures in the elderly. ANZ J Surg 2019;89:E428-32.

18. Knirk JL, Jupiter JB. Intra-articular fractures of the distal end of the radius in young adults. J Bone Joint Surg Am 1986;68:64759.

19. Leone J, Bhandari M, Adili A, McKenzie S, Moro JK, Dunlop RB. Predictors of early and late instability following conservative treatment of extra-articular distal radius fractures. Arch Orthop Trauma Surg 2004;124:38-41.
20. Alluri R, Longacre M, Pannell W, Stevanovic M, Ghiassi A. Volar, intramedullary, and percutaneous fixation of distal radius fractures. J Wrist Surg 2015;4:292-300.

21. Berglund LM, Messer TM. Complications of volar plate fixation for managing distal radius fractures. J Am Acad Orthop Surg 2009;17:369-77.

22. Cai L, Zhu S, Du S, Lin W, Wang T, Lu D, et al. The relationship between radiographic parameters and clinical outcome of distal radius fractures in elderly. Orthop Traumatol Surg Res 2015;101:827-31.

23. Perugia D, Guzzini M, Civitenga C, Guidi M, Dominedò C, Fontana $\mathrm{D}$, et al. Is it really necessary to restore radial anatomic parameters after distal radius fractures? Injury 2014;45:S21-6.

24. Daneshvar P, Chan R, MacDermid J, Grewal R. The effects of ulnar styloid fractures on patients sustaining distal radius fractures. J Hand Surg Am 2014;39:1915-20.

25. Lyu JM, Lin XY, Lin JH. [Risk factors of radius shortening in adult with distal radius fracture after conservative treatment]. Zhongguo Gu Shang 2017;30:513-17. [Article in Chinese] 